\title{
Genitourinary medicine and The Internet
}

\author{
R K W Lau
}

The Internet is now regarded as a rapid and cost-effective means of disseminating information world-wide. As biomedical resources become increasingly available on the Internet, it will prove an invaluable aid to undergraduate education, clinical research, and continuing medical education. It will also provide members of the public direct access to a vast range of medical information. This column aims to provide readers of Genitourinary Medicine with a list of Internet sites and facilities relevant to the pursuit of their clinical and research interests in genitourinary and HIV medicine. It should also be of interest to a wider readership wishing to know more about sexual health issues and where and how this information can be retrieved on the Internet.

Much of the interest relating to the Internet derives from the facilities available through its uniform user-friendly interface, the World Wide Web (WWW, the Web). The Web makes full use of extensive inter-network connectivity through hypertext links, allowing a thread of related information to be electronically linked into a pathway chosen by the user. Web browsers, the programs which provide the "front end" for accessing the Web, can download files from the Internet, allow connection to other Internet facilities such as gopher and ftp (file transfer protocol), print out Web pages, and place bookmarks of Web site addresses in a directory for future retrieval. This obviates the necessity of retyping the Web address each time the site is accessed. The latest Browsers such as Netscape v2.0 support extensions which specify a background to Web pages, background downloading and Web security features which are likely to be the prelude to secure financial transactions on the Internet. Readers wishing to learn more about connecting to and using the facilities on the Internet, related terminology and its medical applications are encouraged to consult the references given below. ${ }^{12}$

This first article lists Web sites dealing in sexually transmitted diseases and safer sex, together with a selection of biomedical journals and Internet search sites. Future papers will deal with sites specific to HIV infection and AIDS, newsgroups, research institutes and additional gopher and ftp addresses for publications on sexually-transmitted diseases and AIDS. Although there will be regular updating, it is hoped that readers will submit lists of Internet sites which they feel may be relevant to the speciality and worthy of mention. Lists of sites from outside the USA would be particularly welcome! Please submit your lists and any comments relating to this column to Dr Richard Lau, Department of Genitourinary Medicine, Beckenham
Hospital, Croydon Rd, Beckenham BR3 3QL, UK; e-mail 100732.1404@compuserve.com.

\section{STD Information}

Healthy Devil Online

http://h-devil-www.mc.duke.edu/h-devil/ An excellent Web site run from Duke University providing useful information on a whole range of health issues for its students, including drugs and alcohol, contraception and safer sex, men's and women's health, sexually transmitted diseases and AIDS.

Ask NOAH about: Sexually transmitted diseases http://www.noah.cuny.edu/stds/stds.html New York Online Health (NOAH) is a public access site run from Columbia University, New York providing information on AIDS and sexually-transmitted diseases. This site is also accessible in Spanish. There are data on New York City public health reports, links to NIH on STD information, pamphlets and brochures on specific STD's and AIDS which can be downloaded, a glossary of AIDS drugs and treatments, and a very good section describing the use of condoms and dental dams.

Department of STD Control, Singapore

http://biomed.nus.sg/dsc/dsc.html

The home page of the Department of STD Control (DSC), Singapore. Describes the history of DSC, its function, personnel, how to use its services with a useful symptom-based description of common sexually-transmitted infections. Good use of graphics.

\section{University of Erlangen Dermatologic Online Image Atlas}

http://www.uni-erlangen.de/docs/FAU/ fakultaet/med/kli/derma/

A resource of dermatovenereological colour images available from this site. Graphicsintensive Web pages mean that downloads tend to be quite slow. Larger images in .jpg format are also available. Lists are in German but a "query interface" allows for a search of text describing the lesion.

\section{Fohns Hopkins Hospital STD Unit}

http://www.jhustd.org/pub/jhustd/

Home page of the Johns Hopkins STD Research Unit, with extensive links to related sites, current research interests, treatment guidelines and patient information.

\section{Safer Sex Information}

Condomania!

http: //www.condomania.com/

A colourful and light-hearted commercial site 
displaying and selling condoms with an educational section on using condoms properly and safer sex.

\section{The Safer Sex Page}

http://www./safersex.org

A comprehensive site dealing with sexual health issues including safer sex and contraception. Has information for health educators.

Yahoo's Safe Sex Index

http://www.yahoo.com/Society_and

Culture/Sex/Safe_Sex/

Yahoo's dedicated safe sex sub-index allows for searches based on keywords and phrases to launch to other sites. Some commercial sites are also listed in these links.

\section{Selected biomedical journals \\ British Medical fournal http: //www.bmj.com/bmj/}

A laudable attempt to publish an electronic version of BMF. Publishes the Journal's current contents, has selected papers as abstracts, a comprehensive index of previous editions and has devoted a large section to its classified job advertisements. Access to the latter requires initial (free) registration.

\section{Fournal of the American Medical Association} http: //ama-assn.org/register/welcome.htm Web site of the American Medical Association and its related journals. Apart from $\mathcal{F} A M A$, it houses the Archives of Dermatology and Archives of Internal Medicine. Access requires initial (free) registration.

Communicable Disease Report, CDSC, UK http://www.open.gov.uk/cdsc/cdschome. htm

Web site of the UK Communicable Disease Surveillance Centre, providing information on its role, departments, list of key personnel with links to other public health institutions in the UK and abroad. Communicable Disease Report (CDR) and CDR Review are available as .pdf files from this site. Like $M M W R$ (below), an Adobe Acrobat Reader is required to read these files. This is provided here.

\section{Morbidity and Mortality Weekly Report, CDC} http: //www.cdc.gov/epo/mmwr/mmwr.html Provides facilities to search by keyword, preview and download current and back issues of $M M W R$ and related publications. Also allows users to be included on their e-mail list for $M M W R$. Adobe Acrobat software is required to view files in .pdf format, but this is available as freeware from Adobe through a licensing agreement with $\mathrm{CDC}$ and obtainable directly from the $M M W R$ web site.

\section{Nature}

http: //www.nature.com

Nature magazine's presence on the Web detailing contents of current and past issues, selected abstracts of important papers and a section devoted to classified job advertisements.

\section{Nature Medicine}

http://medicine.nature.com/

Similar to its sister journal (above), with summaries of contents of its current and previous editions.

\section{New England fournal of Medicine}

http: //www.nejm.org/

Web site of this prestigious medical journal. Updated weekly in parallel with the printed version, it provides a comprehensive index of papers and articles published in its past and current editions. Articles can be browsed and printed, and full-length reprints of papers purchased from this site.

\section{Science}

http://science-mag.aaas.org/science/ Similar in content to Nature with sections dealing with new products, career opportunities and Internet-specific interactive projects.

\section{Medline Searches}

National Library of Medicine (NLM)

http: //www.nlm.nih.gov/

Home page of NLM with extensive links to its services, such as its premier Medline service (subscription required), Medlars (medical literature and retrieval services), Biotechseek (bibliographic citations from selected biotechnology journals), Cancerlit (oncology bibliography), Histline (history of medicine bibliography) and other publications. NLM also holds information on drugs currently being evaluated in AIDS clinical trials (AIDSDRUGS), an extensive AIDS information bibliographic citation index (AIDSLINE) with meeting abstracts from international conferences and a descriptive file of AIDS clinical trials (AIDSTRIALS).Try this selection of freely accessible NLM databases:

\section{AIDS Databases}

http://wwwindex.nlm.nih.gov/databases/ databases_by_subject.html\#aids

\section{HyperDOC}

http://wwwindex.nlm.nih.gov/index/

index.html

Facility for searching medical articles on Web, gopher and ftp servers of the NLM. It is not an index to published medical articles.

Molecular Biology

http://www2.ncbi.nlm.nih.gov/medline/ query form.html

\section{Internet search tools}

Access any of these sites to initiate a more general search, based on a keyword or subject. If a search is unsuccessful or unsatisfactory, you should point to the other search tools. These are usually given at the bottom of a search summary, but can be accessed directly. 
Alta Vista

InfoSeek

Inktomi

Lycos

Yahoo http://altavista.digital.com http://www.infoseek.com http://www.inktomi.com http://lycos.cs.cmu.edu/ http://www.yahoo.com
Yellow Pages http://yellow.com/

RL wishes to thank CompuServe UK for providing free access to its Internet gateway.

1. Pallen $M$. Introducing the Internet. $B M F 1995 ; 311: 1422-4$. 2. Pallen M. The world wide web. BMF 1995;311:1552-6. 